\title{
Preparation and properties of melt-processed thick films of YBCO
}

\author{
A BAILEY, G ALVAREZ, G J RUSSELL and K N R TAYLOR \\ Advanced Electronic Materials, Physics. University of New South Wales, P.O. Box 1, \\ Kensington, N.S.W. 2033, Australia

\begin{abstract}
A detailed study has been carried out of the successful fabrication of high current capacity, thick films of YBCO on yttria-stabilized zirconia substrates. This has involved the optimization of a number of processing parameters including material composition, processing temperatures and times, ink additives and so on.

As a result of this work, thick films have been obtained with thicknesses of as little as $7 \mu$, which are superconducting with $T_{\mathrm{c}}=91.5 \mathrm{~K}$ and have critical current densities at $77 \mathrm{~K}$ of almost $3 \times 10^{3} \mathrm{Acm}^{-2}$. This current decreases rapidly with increasing magnetic field strength, reaching an almost constant value of $100-200 \mathrm{Acm}^{-2}$ at $0.4 \mathrm{~T}$.
\end{abstract}

Keywords. High temperature superconductors; YBCO; thick films; melt processing.

\section{Introduction}

Since the original discovery of the high temperature superconductors, it has been clear that one of the major technological areas likely to benefit from their use will be the field of electronics and microelectronic circuitry. While the logic devices based on Josephson junction effects will almost certainly be fabricated from thin films obtained by one of the many available routes, a vast range of applications will require thin conducting tracks formed from superconducting layers which are capable of carrying significant currents $(1-5 \mathrm{~A})$ in the presence of modest magnetic field strengths. These applications include both passive and active components and it is to be expected that these will be fabricated using techniques which are fully compatible with normal circuit board and microstrip technology.

These requirements are most likely to be solved by the use of thick film superconductors, prepared by screen-printing or spraying specially developed inks onto ceramic substrates and subsequently processed through a thermal cycle based on normal sintering methods. Ideally, these substrates will have low dielectric loss at high frequencies and at present the preferred material would be the high density alumina ceramic substrates which are in common use in the electronics industry.

The conducting tracks, or devices will be formed on these layers either by the use of masks or by lift-off or laser profiling techniques once the superconducting layers are processed. To be compatible with other structures on the substrate, uniform layer thicknesses of $<40 \mu$ will be required which are also smooth and homogeneous.

In view of the importance of such films, a wide range of fabrication techniques have been investigated throughout the world in the past three years. In this work, it has been common to find extensive substrate interactions during processing, superconductor poisoning for thicknesses less than $100 \mu$ and for greater thickness the film has poor mechanical properties. Finally, the generally low critical current densities are depressed below acceptable levels by extremely small magnetic field strengths.

As part of a research programme involving the production of high quality, commercially useful high temperature superconducting materials, we have recently been investigating the preparation of such thick films. This work has led to the 
development of a melt-processing route for YBCO on yttria-stabilized zirconia (YSZ) which leads to high critical current densities in thin, smooth, easily profiled films. The details of this study are outlined in the following.

\section{Experimental}

\subsection{Material processing}

The inks used in this work were prepared from precursor $\mathrm{YBCO}$ powders formed from commercially available $\mathrm{Y}_{2} \mathrm{O}_{3}, \mathrm{BaCO}_{3}$ and $\mathrm{CuO}$. The powders were processed as for normal superconducting material by a double thermal treatment involving a reaction and a sintering cycle at $900^{\circ} \mathrm{C}$. We found that these processing conditions affected the final quality of the films, and various reaction/sinter times were investigated. These varied from $2 \times 6(6$ reaction, 6 sinter $)$ to $2 \times 48 \mathrm{~h}$ periods at $900^{\circ} \mathrm{C}$ with relatively slow cooling to room temperature. The processed bulk YBCO was ground and sieved to obtain an average particle size of approximately $0.5 \mu$. The inks were then prepared from these powders by ultrasonically mixing in triethanolamine to obtain the desired viscosity. In the present work, the films were then prepared by the use of a doctor blade passed over a layer of the ink which had been painted onto the substrate. The layers obtained this way were up to $40 \mu$ thickness and were dried in air at $200^{\circ} \mathrm{C}$ for 20 minutes.

Both alumina and YSZ substrates were used in the original studies; however it rapidly became clear that the film-substrate interactions with alumina led to severe degradation and poisoning effects which have been reported by many other workers, and the remainder of the work was restricted entirely to thin, YSZ substrates.

Once dry, the films were processed rapidly at an elevated temperature using the cycle outlined in table 1.

A preliminary study showed that for firing temperatures lying below the peritectic temperature $\left(1000^{\circ} \mathrm{C}\right.$ in air), the resulting films were highly granular, with poor superconducting properties, while firing above the peritectic the films led to considerable texturing and improved critical currents in agreement with the findings of Shields et al (1989). In the present work, for $T_{f}=1015^{\circ} \mathrm{C}$ (i.e. $15^{\circ} \mathrm{C}$ above the peritectic), the films could be seen to have extensive areas of crystallization with clear evidence for a liquid phase process having occurred, as might be expected.

Because of the known advantages to be gained in bulk material by the addition of silver to the powder before sintering, we also studied the effects of silver addition on the final superconducting parameters, using the same processing conditions. These advantages include enhanced grain growth (Baliga and Jain 1989), improved critical

Table 1. Film processing cycle

\begin{tabular}{lc}
\hline Heat to $900^{\circ} \mathrm{C}$ & $5^{\circ} \mathrm{C} / \mathrm{min}$ \\
Soak at $900^{\circ} \mathrm{C}$ & $10 \mathrm{~min}$ \\
Heat to firing temperature $T_{f}$ & $4^{\circ} \mathrm{C} / \mathrm{min}$ \\
Hold at $T_{f}$ & $5 \mathrm{~min}$ \\
Cool to room temperature & $2^{\circ} \mathrm{C} / \mathrm{min}$ \\
The entire cycle was carried out in air.
\end{tabular}


currents (Dwir et al 1989) and reduced intergranular resistivities (Pavuna et al 1988), all of which could contribute significantly to the performance of thick film materials.

\subsection{Results}

X-ray diffraction from the surfaces of the processed films showed that for all types of powder there was significant texturing of the surface layer, with the $c$-axis normal to the film plane as may be seen from figure 1 , where the relative intensity of the axial $(001)$ lines can be observed to increase after processing. The intensity ratio $F$ of the $(005) /(103$ +110 ) lines can be used as a simple figure of merit to describe the degree of texture. This texturing became particularly marked for the $2 \times 24+10 \mathrm{wt} \% \mathrm{Ag}$ film where $F$ increased by an order of magnitude over that of the films without silver and a factor of 40 above a randomly-oriented powder. The measured thickness of the films after processing decreased slightly with the normal YBCO inks, although since it is known that a barrier layer based on $\mathrm{BaZrO}_{3}$ forms during processing (Wellhofer et al 1989) the actual thickness of the superconducting layer is only of the order of $20 \simeq 30 \mu$. In the case of silver-containing films, however, the final processed film thickness lay in the range $7-10 \mu$, the free surfaces being extremely smooth and uniform.

Measurements of the electrical characteristics using a conventional 4-point resistivity system with silver paste electrodes showed that with increased processing time the sharpness of the superconducting transition at $91.5 \mathrm{~K}$ is optimized for the $(2 \times 24)$ hour cycle as shown in figure 2 . The normal state resistivity (at $100 \mathrm{~K}$ ) also passes through a broad minimum under these conditions and the critical current density reaches a maximum value close to $1000 \mathrm{Acm}^{-2}$ as may be seen from figure 3 . For the pure YBCO films there was a considerable range of measured critical current densities which appeared from both optical and SEM microscopy, to be associated with the distribution of cracks in the film surface.

Addition of silver to the superconducting powder used for the manufacture of ink, dramatically improves the performance of the films. As may be seen from figure 1, the texturing figure of merit increases rapidly once silver is added, and the variation of $F$

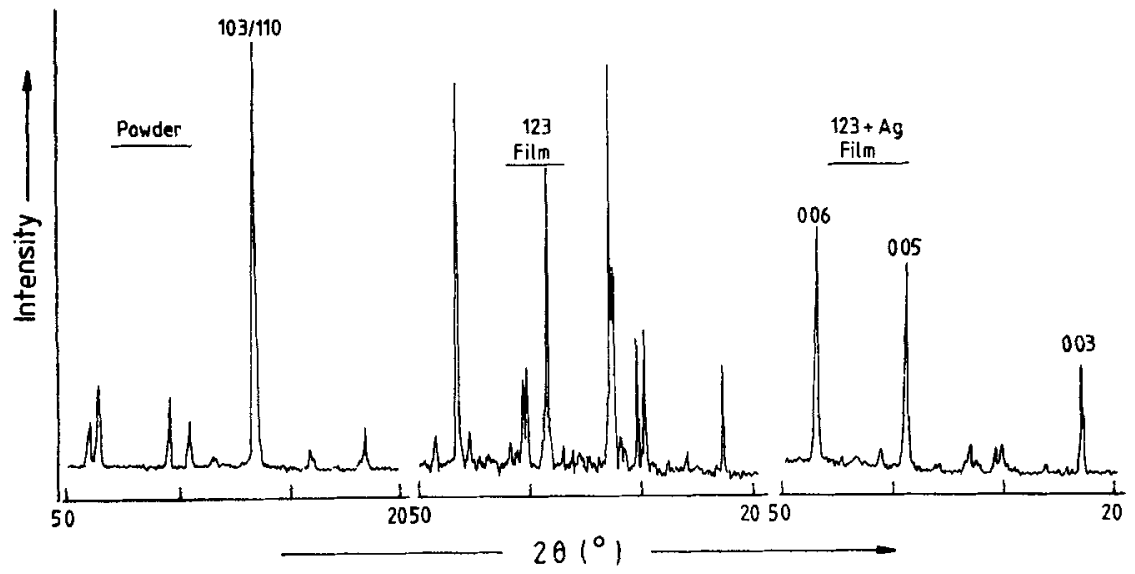

Figure 1. X-ray powder diffraction patterns from a randomly-oriented powder, the surface of a (123) film and a (123) $+10 \mathrm{wt} \% \mathrm{Ag}$ film, showing the development of texture. 


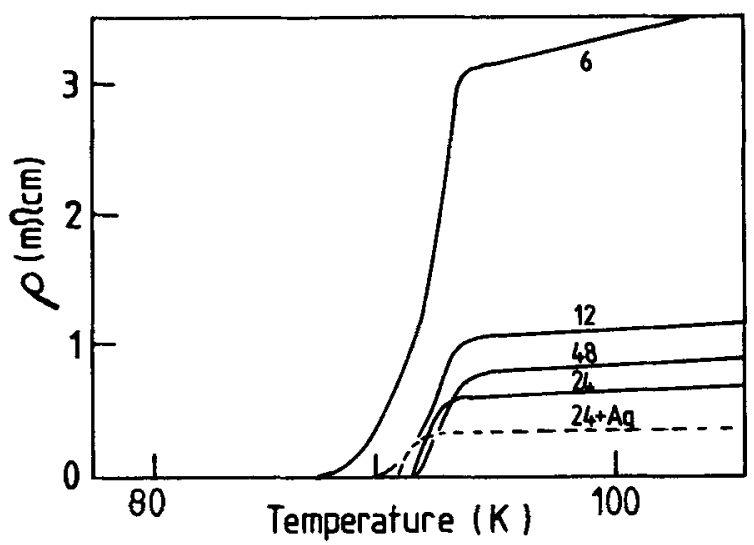

Figure 2. Effect of precursor powder-processing time on the resistive transition. The transition for a $10 \mathrm{wt} \% \mathrm{Ag}$ film is also shown.

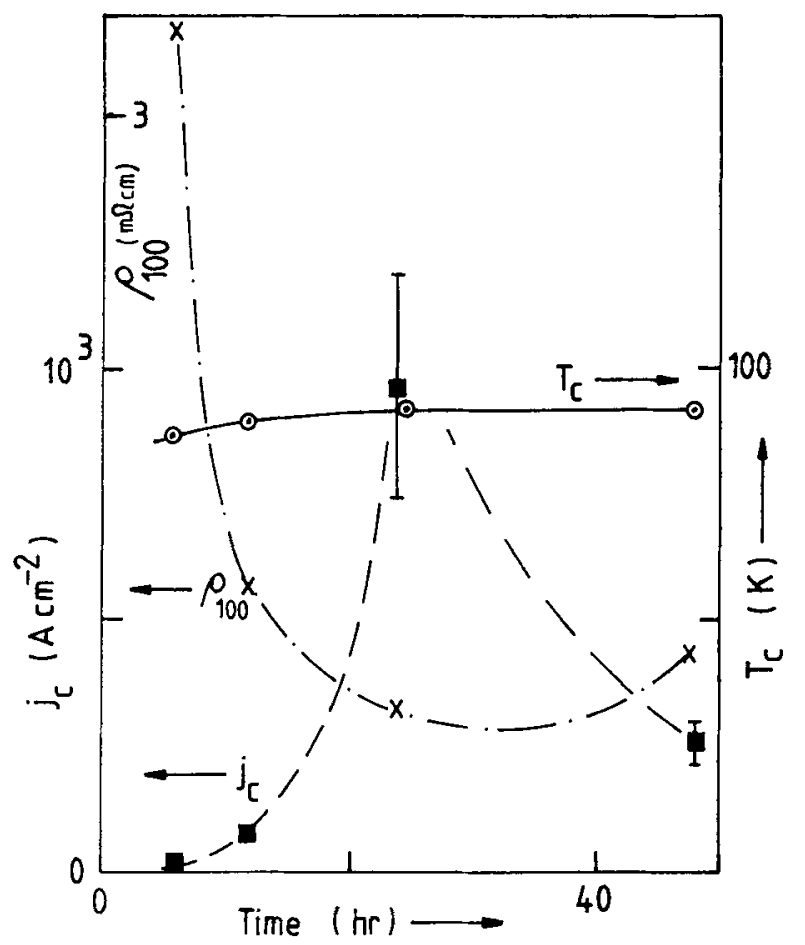

Figure 3. Variation of the normal state resistivity, the critical current density and the critical temperature with powder-processing time. The times shown are the periods of each firing i.e. $24 \mathrm{~h}=2 \times 24$ sample.

with silver concentration is shown in figure 4 , where even at $10 \mathrm{wt} \% \mathrm{Ag}$ the degree of texturing is still increasing rapidly. The films prepared this way show evidence for more liquid formation than was the case without silver, an effect presumably connected with the fact that silver is molten at the processing temperature and undoubtedly affects the thermodynamics of the system. Electrical measurements showed that the addition of 


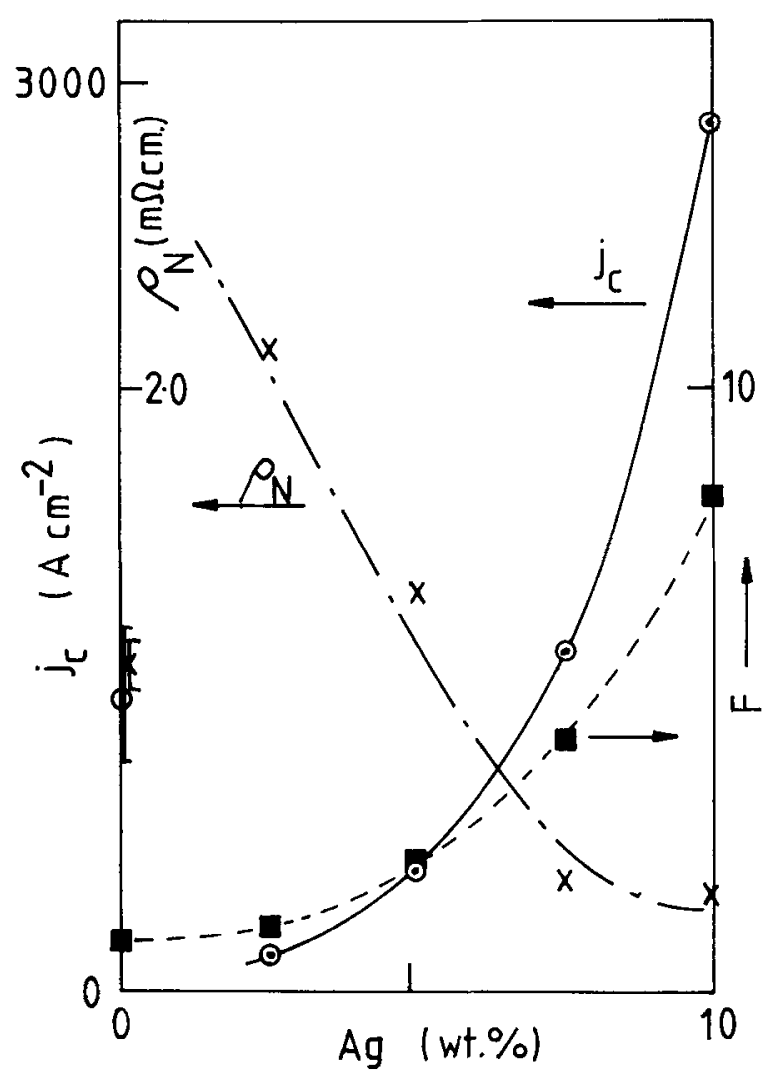

Figure 4. Variation of the texture figure of merit $F$, the normal state $(300 \mathrm{~K})$ resistivity and the critical current density as a function of silver concentration.

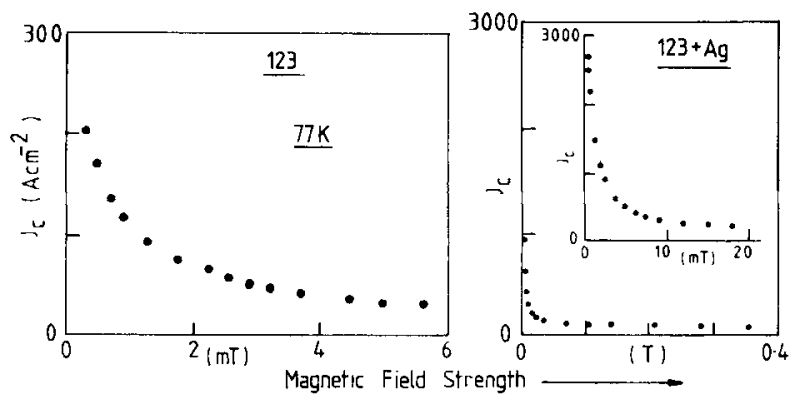

Figure 5. Magnetic field dependence of the critical current density for both types of film.

silver also led to a decrease in the normal-state resistivity and a large increase in the critical current density at $77 \mathrm{~K}$ to almost $3 \times 10^{3} \mathrm{Acm}^{-2}$. The dependence of both of these parameters with silver concentration is shown in figure 4 . The overall quality of the films also appears to be improved under these conditions since there is far less spread of the values of these parameters from specimen to specimen.

The effects of an applied magnetic field on the critical current densities are shown in figure 5, where the rapid decrease caused by intergranular effects can be seen for both 

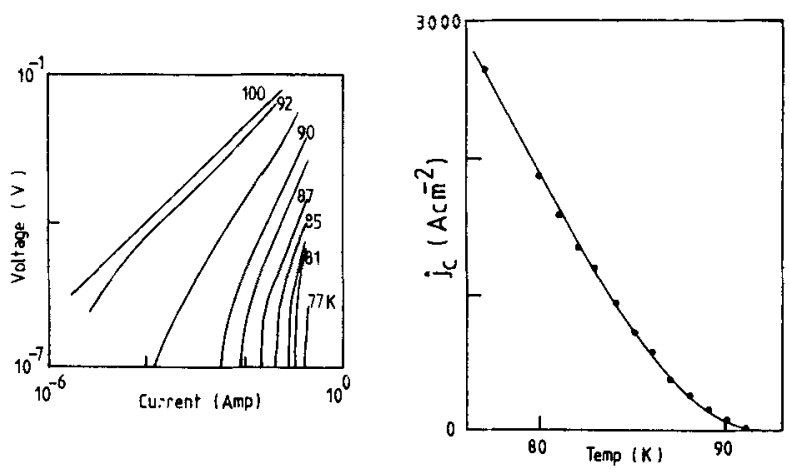

Figure 6. The temperature dependence of the $I-V$ characteristics of a silver-containing film and the variation of the critical current density at $10^{-6} \mathrm{~V}$ for a specimen of cross-section $7 \mu \times 2 \mathrm{~mm}$.

types of film. It should be noticed, however, that with silver addition, the critical current density reaches a plateau level of approximately $100 \mathrm{Acm}^{-2}$ above about $0.05 \mathrm{~T}$ which remains to the highest fields used in this study. The temperature dependence of the current-voltage characteristics of the $10 \mathrm{wt} \% \mathrm{Ag}$ film is shown in figure 6 as also is the variation of the critical current density derived from this data.

\section{Conclusions}

The performance of these thick films, prepared by melt-processing techniques, shows that high quality material with useful superconducting properties can be prepared by careful optimization of the processing parameters. For pure YBCO films, the improvements brought about by extensive crystallization and texturing are assisted by the development of the apparently inert barrier layer based on barium zirconate which is produced during processing. Unfortunately however, inter- and transgranular cracking restricts the reproducibility of the film behaviour. Once silver is added to the inks used to prepare the films the surface morphology improves, the critical current densities reach acceptable levels and the variation in film quality is greatly reduced.

\section{References}

Baliga S and Jain A L 1989 Appl. Phys. A. (Preprint)

Dwir B, Affronte M and Pavuna D 1989 Appl. Phys. Lett. 55399

Pavuna D, Berger H, Affronte M, van der Maas J, Capponi J J, Guillot M and Tholence J L 1988 Solid State Commun. 68535

Shields T C, Wellhofer F, Abell J S, Taylor K N R and Holland D 1989 Physica C (to be published)

Wellhofer F, Shields T C, Abell J S and Taylor K N R 1989 MRS Fall Meeting, Boston(to be published) 